\title{
Analysis of Multi Resolution Image Denoising Scheme using Fractal Transform
}

\author{
B. Chinna Rao 1, Dr. M. Madhavi Latha 2 \\ 1 Department of ECE, RK College of Engineering, Vijayawada, A.P, INDIA. \\ Chinnarao_ece@yahoo.co.in[1] \\ 2 Department of ECE, JNTU College of Engineering, Hyderabad, A.P, INDIA. \\ mlmakkena@yahoo.com[2]
}

\begin{abstract}
:
For communication and storage efficiency, image data should be substantially Compressed. The compression ratio is limited by noise, which degrades the correlation between pixels. Noise can occur during image capture, transmission or processing, and may be dependent on or independent of image content. This work proposes a novel algorithm which reduces noise in colour images. Simulation results proved that $86 \%$ efficiency has been achieved, while considering 415 pixels.
\end{abstract}

\section{Keywords}

Image Denoising, Wavelet transform, Fractal Transform, Fractal coding. Fractal Thresholding, Wavelet Thresholding

\section{Introduction}

The appearance of the wavelet filtering has been applied in image denoising for many years. While the performance is not very significant. The wavelet transform is a linear operator, Wavelet signal denoising appears to be a natural frame. The use of wavelet decomposition for noise decomposition is often performed in the image logarithm area. With an acceptable quality, appropriate error concealment techniques dealing with shape and texture data are necessary. Here, a main shortcoming of transformation is that it is based on estimation of the information of original image[1]. In order to deal with multiplicative noise model mentioned above, we attempt to reduce speckle in images using multi-Resolution decomposition[2-8]. For one-dimensional piecewise smooth signals, wavelets has been established as a powerful tool. However, nature images always contain abundant geometric characteristics, such as edges, contours and textures, etc. It is believed that wavelets can poorly handle the directional information of the natural images, and cannot provide the optimal sparse representation of nature images [5]. Recently, several more effective transforms with good directionality and anisotropy are proposed, such as ridgelet transform[3], curvelets transform [4],contourlet Transform(CT) [5] and wavelet-based contourlet transform(WCT) [8], etc. The directionality and anisotropy characteristics enable all these newly proposed transforms in a certain degree to capture the directional information of a natural image into subbands of different scale and frequency.Thus, these transforms can make full use of the directional information to distinguish the important image features from the nondirectional noise. An image is often corrupted by noise in its acquisition and transmission. Image denoising is used to remove the additive noise while retaining as much as possible the important signal features. In recent years many approaches to image denosing have been proposed [1]-[6], some of them are based on single wavelet and the others based on multiple wavelets. Single wavelet and multiple wavelets have their own advantages and limitations. For instance, multiple wavelets can possess simultaneously orthogonality, symmetry, and short support, while a single wavelet cannot possess all these properties at the same time. So multiple wavelets is much more 
flexible than single wavelet, however, single wavelet is the foundation of the multiple wavelets. The member of single wavelet family is much more abundant than multiple wavelets, and it is easy to calculate. The basic approach based on wavelet transform (single wavelet transform and multiple wavelets transform) consists of the following three steps:

1) Compute the WT coefficients of the signal;

2) Perform some specified processing on these coefficients;

3) Compute the inverse WT to obtain the processed image.

Different wavelet methods are differentiated by the step 2. Since the work of Donoho and Johnstone [1][2], many methods based on WT were proposed. These approaches can be classified by the selection of wavelet or threshold. This paper gives a comparison between single wavelet and multiple wavelets in image denosing.Another problem seen in the image denoising techniques is the pseudo-Gibbs artifacts which occur due to the thresholding of some of the transform coefficients to zero. Sometimes artifacts cannot be permitted in result images. For example, in medical image processing, artifacts can bring mistake in diagnosis. These artifacts can be reduced by nonlinear diffusion [12]. Denoising by combining wavelet transform and anisotropic diffusion to reduce these artifacts has been explored before and shown to give positive results [13]. Recently, denoising by combining Fractlet shrinkage and non linear diffusion has been implemented and the results show significant reduction in the artifacts [14]. In this paper, we combine anisotropic diffusion along with our hybrid denoising method which involves the Fractlet transform and the wavelet transform. We also compare the results obtained by our method with those obtained by Fractals based denoising and wavelet based denoising methods.

\section{Wavelet Transform}

Wavelet transform (WT) represents an image as a sum of wavelet functions with different locations and scales [17].Any decomposition of an image into wavelets involves a pair of waveforms: one to represent the high frequencies corresponding to the detailed parts of an image (wavelet function) and one for the low frequencies or smooth parts of an image (scaling function).
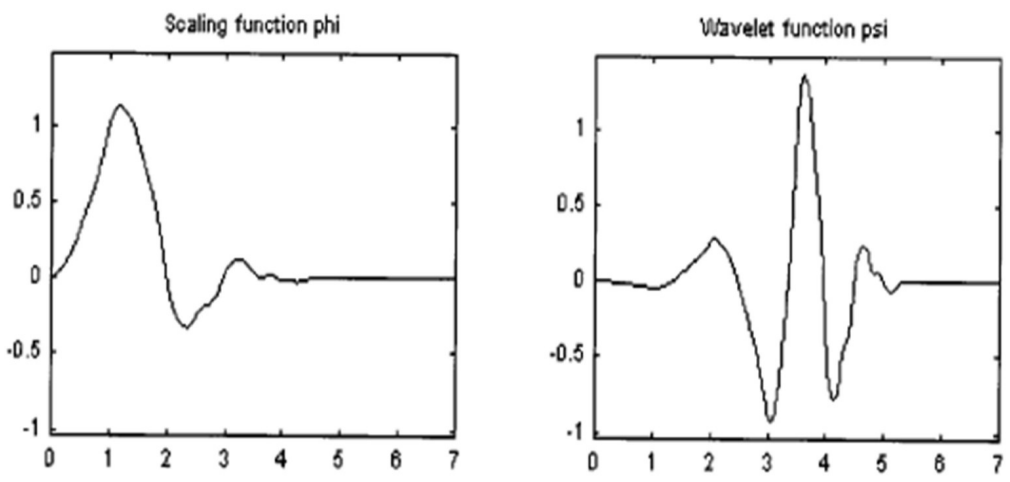

Fig.1.Wavelet Transform.

Fig. 1 shows two waveforms of a family discovered in the late 1980s by Daubechies: the right one can be used to represent detailed parts of the image and the left one to represent smooth parts of the image. The two waveforms are translated and scaled on the time axis to produce a 
set of wavelet functions at different locations and on different scales. Each wavelet contains the same number of cycles, such that, as the frequency reduces, the wavelet gets longer. High frequencies are transformed with short functions (low scale). Low frequencies are transformed with long functions (high scale). During computation, the analyzing wavelet is shifted over the full domain of the analyzed function. The result ofWTis a set of wavelet coefficients, which measure the contribution of the wavelets at these locations and scales.

\subsection{Multi resolution Analysis}

Fractal $\mathrm{T}$ performs multi resolution image analysis [18]. The result of multiresolution analysis is simultaneous image representation on different resolution (and quality) levels [19]. The resolution is determined by a threshold below which all fluctuations or details are ignored. The difference between two neighboring resolutions represents details. Therefore, an image can be represented by a low-resolution image (approximation or average part) and the details on each higher resolution level. Let us consider a one-dimensional (1-D) function $\mathrm{f}(\mathrm{t})$. At the resolution ' $\mathrm{j}$ ' level , the approximation of the function. The intuition behind using lossy compression for denoising may be explained as follows. A signal typically has structural correlations that a good coder can exploit to yield a concise representation. White noise, however, does not have structural redundancies and thus is not easily compressible. Hence, a good compression method can provide a suitable model for distinguishing between signal and noise. The discussion will be restricted to wavelet-based coders, though these insights can be extended to other transformdomain coders as well. A concrete connection between lossy compression and denoising can easily be seen when one examines the similarity between thresholding and quantization, the latter of which is a necessary step in a practical lossy coder. That is, the quantization of wavelet coefficients with a zero-zone is an approximation to the thresholding function (see Fig. 1). Thus, provided that the quantization outside of the zero-zone does not introduce significant distortion, it follows that wavelet-based lossy compression achieves denoising. With this connection in mind, this paper is about wavelet thresholding for image denoising and also for lossy compression. The threshold choice aids the lossy coder to choose its zero-zone, and the resulting coder achieves simultaneous denoising and compression if such property is desired

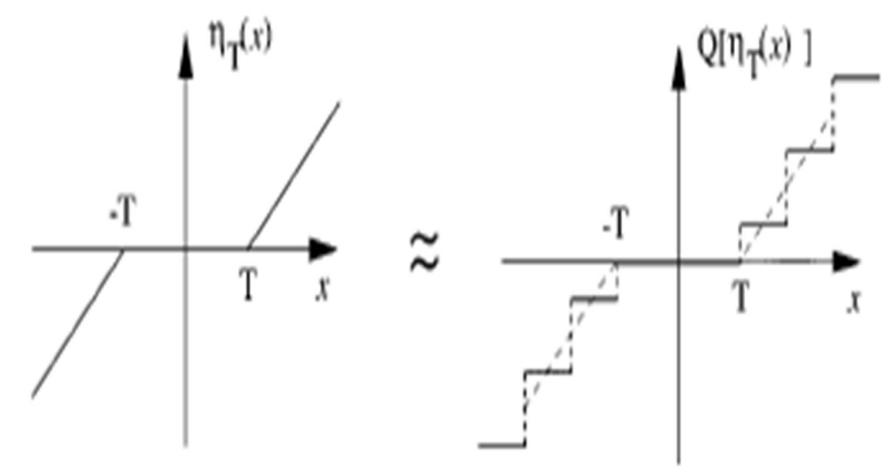

Fig. 2. Thresholding function can be approximated by quantization

The theoretical formalization of filtering additive iid Gaussian noise (of zero-mean and standard deviation ) via thresholding wavelet coefficients was pioneered by Donoho and Johnstone [14]. A wavelet coefficient is compared to a given threshold and is set to zero if its magnitude is less than the threshold; otherwise, it is kept or modified (depending on the thresholding rule). The threshold acts as an oracle which distinguishes between the insignificant coefficients likely due to noise, and the significant coefficients consisting of important signal structures. Thresholding rules are especially effective for signals with sparse or near-sparse representations where only a 
small subset of the coefficients represents all or most of the signal energy. Thresholding essentially creates a region around zero where the coefficients are considered negligible. Outside of this region, the thresholded coefficients are kept to full precision (that is, without quantization). Their most well-known thresholding methods include VisuShrink [14] and SureShrink [15]. These threshold choices enjoy asymptotic minimax optimalities over function spaces such as Besov spaces. For image denoising, however, VisuShrink is known to yield overly smoothed images.

Since the works of Donoho and Johnstone, therehas been much research on finding thresholds for nonparametric estimation in statistics. However, few are specifically tailoredfor images. In this paper, we propose a framework and a near-optimal threshold in this framework more suitable for image denoising. This approach can be formally described as Bayesian, but this only describes our mathematical formulation, not our philosophy. The formulation is grounded on the empirical observation that the wavelet coefficients in a subband of a natural image can be summarized adequately by a generalized Gaussian distribution (GGD) To achieve simultaneous denoising and compression, the nonzero thresholded wavelet coefficients need to be quantized. Uniform quantizer and centroid reconstruction is used on the GGD. This criterion balances the tradeoff between the compression rate and distortion, and yields a nice interpretation of operating at a fixed slope on the rate-distortion curve.

\section{Fractal Transform}

Originally, fractal-based methods sought to express a target set as a union of shrunken copies of itself. However, most real-world images are rarely so entirely self-similar. Instead, selfsimilarity may be exhibited only locally, in the sense that subregions of an image may be selfsimilar. This is the basis of the block-based fractal encoding scheme introduced by Jacquin [11]. Most fractal-based image coding methods are based on this scheme, which can be outlined as follows:

1) As illustrated in Fig. 1, the image is subdivided into two different non-overlapping partitions of sub-blocks:

a) $\mathrm{M}_{-} \mathrm{M}$ domain(parent) blocks, Di. For instance, when the image is square with a power of size, we choose $M=2 \mathrm{~m}$, for some integer $\mathrm{m}>1$

b) $\mathrm{N}$ _ $\mathrm{N}$ domain (child) Ri. Typically we choose $\mathrm{N}=2 \_\mathrm{M}$, so that the size of a parent block is four times of a child block.

2) Each child block, $\mathrm{Ri}, \mathrm{k}=1,2,3 \ldots . . \mathrm{N} 2$, is then matched to its most 'similar' parent block for the same 
The International journal of Multimedia \& Its Applications (IJMA) Vol.2, No.3, August 2010

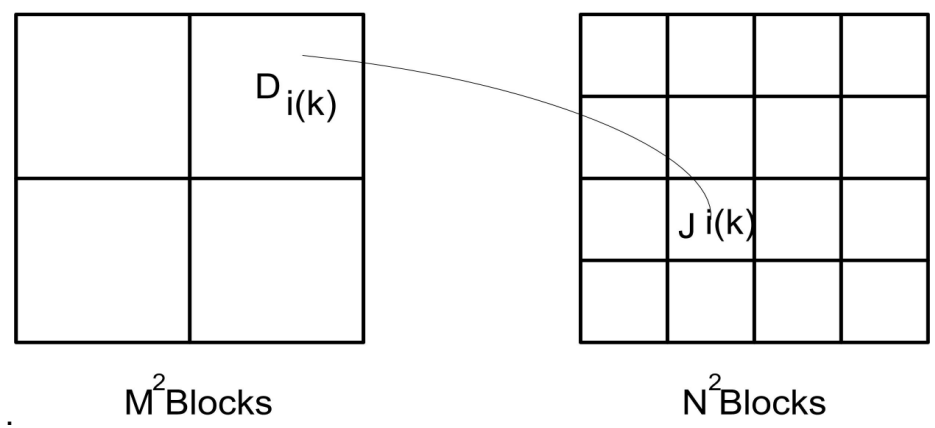

Fig.3. Uniform image partitioning for fractal image coding.

3) The similarity between the parent block $\mathrm{Di}(\mathrm{k})$ and the child block Rk, is in the sense that the subimage on the parent block $\mathrm{Di}(\mathrm{k})$ can be transformed "closely" to the subimage on the child block Rk via a contractive mapping. This contractive transformation is a composition of a geometric mapping wi(k) followed.

4) A least-squares fit is then performed between the child block $\mathrm{Rk}$, and the geometrically transformed parent block using affine grey-level mapping Rk.

$$
\varphi(t)=\alpha t+\beta
$$

\subsection{Fractal Decoding}

The fractal decoding algorithm is a fast and recursive process which can be summarized as follows: Starting with any initial image (typically a blank image), each range subblock Rk, is predicted from its fractal code. This process is then repeated recursively until a desirable convergence is achieved. Convergence may be defined in terms of the difference between two consecutive iteration estimates. Next, we outline the fractal-based joint image denoising and resizing scheme.

\subsection{Fractal Image Denoising}

In [12], a simple and effective fractal-based strategy for smoothing noisy images in the pixel as well as the wavelet domains of the noisy image was proposed. First, it was observed that straightforward fractal coding of a noisy image yields some degree of noise reduction. This may be explained by the fact that self-similar structures found in natural images are generally reconstructed rather well through fractal coding whereas the noisy components cannot be approximated well in this way. We have also shown that one can achieve better image denoising results by estimating the fractal code of the original noiseless image from that of the noisy observation. From this predicted fractal code, one can generate a fractally denoised estimate of the original image. Due to space limitation, the details of this fractal-based image denoising scheme are not included here. The reader is referred to our previous work in [13, 14].

\subsection{Fractal Image Interpolation}

In order to increase the size of the image by a power of two (in each direction), a simple fractal strategy can be applied [15]. This method is performed entirely during the fast converging fractal decoding process. For instance, for the commonly used test image of "Lena" of size 512 X 512 
and suppose we wish to double the size of this test image to obtain a fractal interpolated image of size 1024 X 1024.

1) First, the original image is fractal encoded, using the standard fractal coding scheme, outlined above. This must be done at a sufficiently high fractal resolution (i.e. small domain/range blocks) in order to achieve a sufficiently high quality fractal representation of the image. For instance for the standard fractal scheme, we use $(\mathrm{M}, \mathrm{N})=(64,128)$ which results in mapping a $8 \mathrm{X}$ 8 pixel parent blocks into a $4 \mathrm{X} 4$ child blocks.

2) Then, in order to double the size of the fractally decoded image, during the decoding process one simply starts with an initial blank image seed of the same size as the desired interpolated image.

This simple fractal-based process results in a fractally interpolated image of size $1024 \mathrm{X} 1024$ Of course, modifying (increase or decreasing) the size of the image by any power of 2 in each direction can be performed in a similar manner, by simply applying the fractal code on an image blank image seed of a suitable size.

\section{Novel Image Denoising Schme}

In fact, Gaussian scheme is the main process behind the proposed scheme. Image smoothing using nonlinear diffusion has been used in the field of image processing. If $u 0$ denote the observed noisy image which is known to be the sum of the original image s and some Gaussian noise $n$

$$
\begin{aligned}
& \qquad u_{0}(x)=s(x)+p(x)-2 q(x), \quad x=\left(x_{1}, x_{2}\right) \in R \\
& \text { We consider the diffusion process } \\
& \qquad \frac{\partial u}{\partial t}=\nabla \cdot(g(|\nabla u|) \nabla u) \\
& \text { with the given noisy signal } u_{0} \text { as the initial condition } \\
& \qquad u(x, 0)=u_{0}(x), \quad x \in R^{2}
\end{aligned}
$$

and periodic boundary conditions. Here, the time $t$ acts as a scale parameter for filtering. The choice of the diffusion constant $\mathrm{g}(\mathrm{x})=$ const corresponds to a strong smoothing of $\mathrm{u}$ with increasing t. typically, $\mathrm{g}(\mathrm{x})$ is a non-negative decreasing function with limit of $\mathrm{g}(\mathrm{x})$ tending to zero as $\mathrm{x}$ approaches infinity. The diffusivity $\mathrm{g}$ controls the smoothing process by admitting strong diffusion if the gradient is small, possibly caused by noise, and by slowing down or even stop the smoothing for large gradients.

One of the serious problems in the diffusion model in (5) is that it is very sensitive to noise. The noise often introduces very large oscillations of the gradient. Therefore, the model in (4) possibly misconstrues the true edges and heavy noise, which leads to undesirable diffusion in regions where there is no true edge. A typical improved model, to remedy these deficiencies, is based on Gaussian regularization

$$
\frac{\partial u}{\partial t}=\nabla \cdot\left(g\left(\nabla\left(G_{\sigma} * u\right)\right) \nabla v-\nabla p\right)
$$

where $G_{\sigma}$ is a Gaussian kernel with variance. The Gaussian filtering acts as a preprocessing to reduce the influence of noise during the diffusion process. The diffusion is applied to the difference image $\mathrm{u} 0 \mathrm{-uc}$, instead of directly applying to the denoised image. Here $\mathrm{u} 0$ is the noisy 
image and uc is the denoised image. After some iteration steps of the diffusion scheme, the smoothened difference image is added to the denoised image to obtain the final result. The idea here is to keep the low-frequency coefficients and the significant coefficients, that is those coefficients whose magnitude is above the threshold, untouched and slightly change the coefficients that have been thresholded to zero in such a way that the image is smoothed.

The idea behind this approach is that while the denoised image contains the important features of the image to be reconstructed, the difference image particularly contains high frequency components which mainly correspond to noise. Applying the diffusion only to the difference image avoids the narrow peaks or textures from getting smoothened too much as in conventional diffusion. We can thus retain the signal amplitude of the detail components while reducing the pseudo- Gibbs oscillations at the same time, in comparison to those methods directly using curvelet shrinkage, As the diffusivity function $\mathrm{g}$.

This section focuses on the estimation of the GGD parameters, $\sigma_{X}$ and ${ }^{\beta}$ which in turn yields a data-driven estimate of $T_{\beta}\left(\sigma_{X}\right)$ that is adaptive to different subband characteristics. The noise variance needs to be estimated first. In some situations, it may be possible to measure based on information other than the corrupted image. If such is not the case, it is estimated from the subband by the robust median estimator, also used in [14], [15], To summarize, we refer to our method as BayesShrink which performs soft-thresholding, with the data-driven, subbanddependent threshold,

$$
\hat{T}_{B}(\hat{\sigma}(X))=\frac{\hat{\sigma}^{2}}{\hat{\sigma}_{X}}
$$

\section{Experimental Results}

The grayscale images goldhill, lena, barbara and baboon are used as test images with different noise levels. The original images are shown in fig 3. The wavelet transform employs Daubechies least asymmetric compactly-supported wavelet with eight vanishing moments [11] with four scales of orthogonal decomposition 

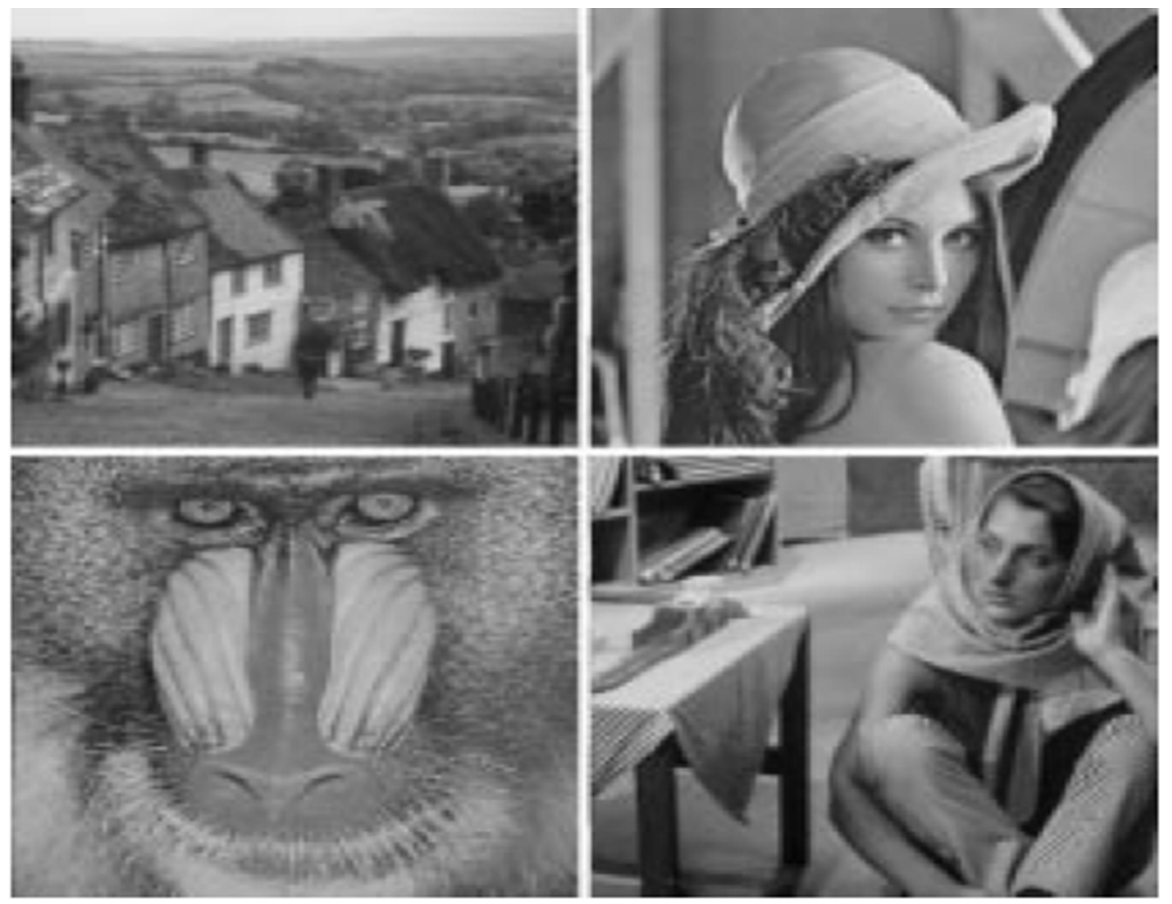

Fig. 4. Original images. From top left, clockwise: goldhill, lena, barbara and baboon

We have also made comparisons with the Wiener filter, the best linear filtering possible. The version used is the adaptive filter, wiener2, in the MATLAB image processing toolbox, using the default settings ( local window size, and the unknown noise power is estimated). The MSE results are shown in Table I, and they are considerably worse than the nonlinear thresholding methods, especially when is large. The image quality is also not as good as those of the thresholding methods. The MDLQ-based compression step introduces quantization noise which is quite visible. As shown in the last column of Table I, the coder achieves a lower bitrate, but at the expense of increasing the MSE. The MSE can be even worse than the noisy observation for small values of , especially for the highly detailed images. This is because the quantization noise is significant compared to the additive Gaussian noise. 
The International journal of Multimedia \& Its Applications (IJMA) Vol.2, No.3, August 2010
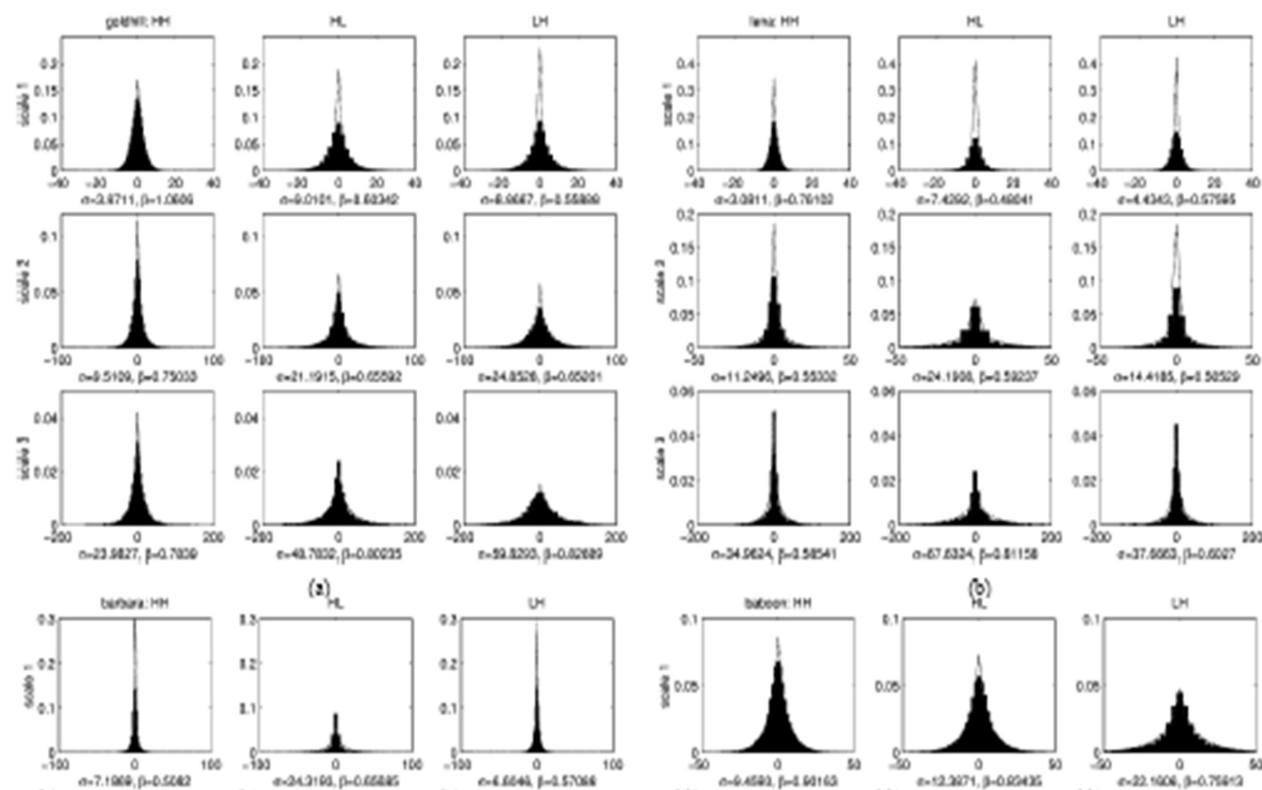

(2)
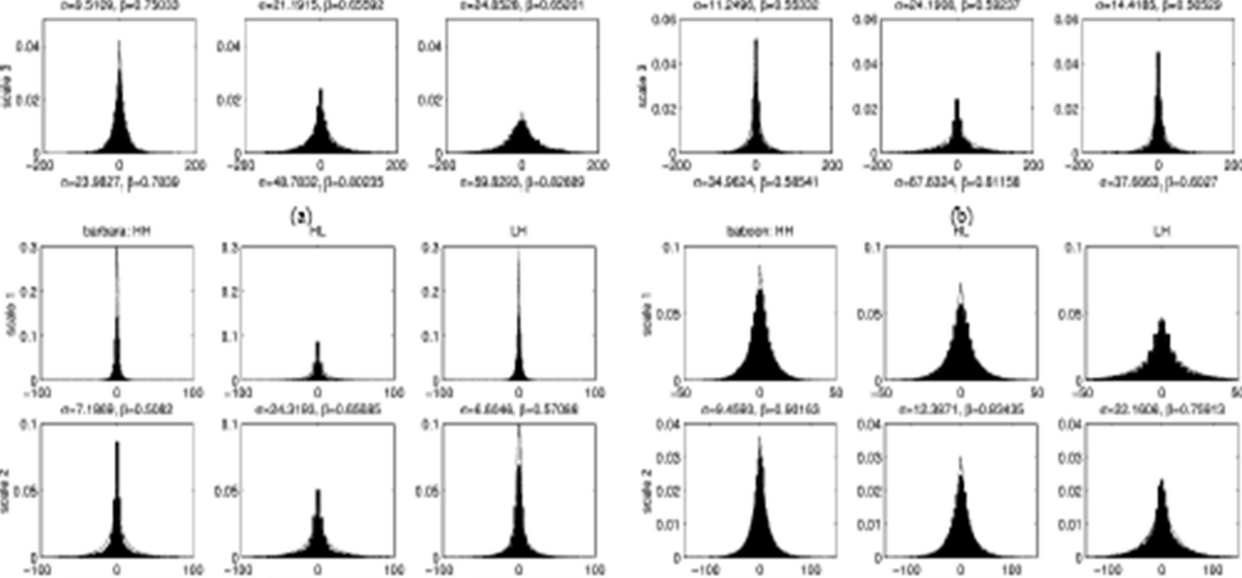

(b)
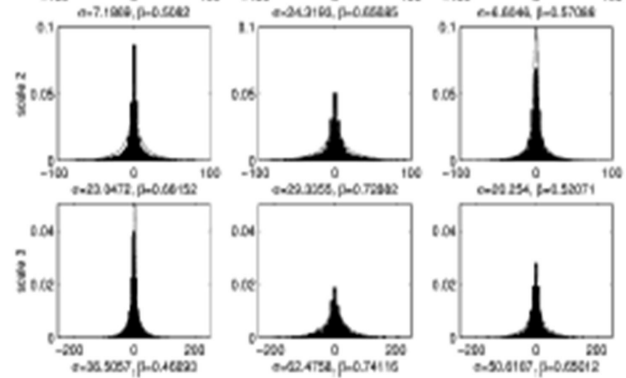

(c)
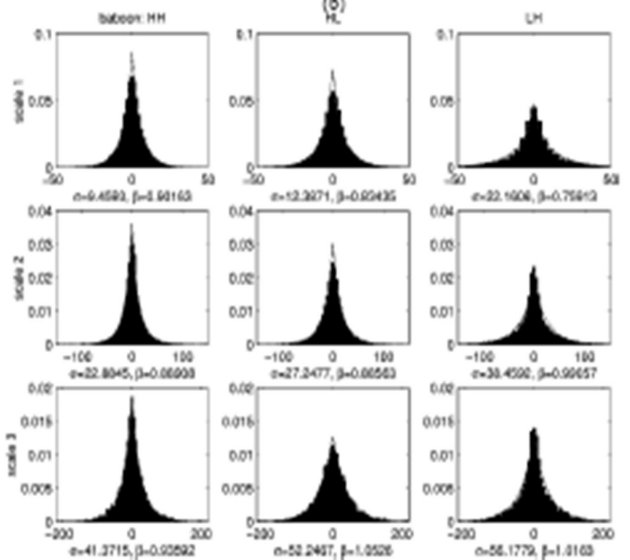

(d)

Fig. 5.Histogram of the wavelet coefficients of four test images.For each image, from top to bottom it is fine to coarse scales: from left to right, they are the HH, HL, and LH subbands, respectively

For larger, the compressed images can achieve noise reduction up to approximately $75 \%$ in terms of MSE. Furthermore, the bitrates are significantly less than the original $8 \mathrm{bpp}$ for grayscale images. Thus, compression does achieve denoising and the proposed MDLQ-based compression can be used if simultaneous denoising and compression is a desired feature. If only the best denoising performance were the goal, obviously using solely BayesShrink is preferred. A fair assessment of the MDLQ scheme for quantization after thresholding is the R-D curve used in Hansen and Yu [17] (see http://cm.belllabs .com/stat/binyu/publications.html). This R-D curve is calculated using noiseless coefficients, and yields the best possible in terms of R-D tradeoff when the quantization is restricted to equal-binwidth. It thus gives an idea on how effective MDLQ is in choosing the tradeoff with respect to the optimal. The closeness of the MDLQ point to this R-D lowerbound curve indicates that MDLQ chooses a good R-D tradeoff without the knowledge of the noiseless coefficients required in deriving this R-D curve. the resulting images of each denoising method for goldhill and (a zoomed-in section of the image is displayed in order to show the details). Table II compares the threshold values for each subband chosen by OracleShrink, SureShrink and BayesShrink, averaged over five runs. It is clear that the BayesShrink threshold selection is comparable to the SURE threshold and to the true optimal threshold. Some of the unexpectedly large threshold values in SureShrink comes from the universal threshold, not the SURE threshold, and these are placed in parentheses in the table. Table II(c) lists the thresholds of BayesShrink, and the thresholds in parentheses correspond to the case when, and all coefficients have been set to zero. Table III tabulates the values of chosen 
The International journal of Multimedia \& Its Applications (IJMA) Vol.2, No.3, August 2010

by for each subband of the goldhill image, , averaged over five runs. The MDLQ criterion allocates more levels in the coarser, more important levels, as would be the case in a practical subband coding situation. A value of indicates that the coefficients have already been thresholded to zero, and there is nothing to code.

\section{Conclusions}

Two main issues regarding image denoising were addressed in this paper. Firstly, an adaptive threshold for wavelet thresholding images was proposed, based on the GGD modeling of subband coefficients, and test results showed excellent performance. Secondly, a coder was designed specifically for simultaneous compression and denoising. The essence of fractal-based denoising, both in the wavelet as well as pixel domains, is to predict the fractal code of a noiseless image from its noisy observation. We have experimentally shown that the fractalwavelet denoising scheme is able, at least for moderate

\begin{tabular}{l|r|r|r}
\hline & \multicolumn{3}{c}{ Orientations } \\
\hline Scales & HH & HL & LH \\
\hline \hline 1 (finest) & 84.90 & 37.36 & 38.04 \\
\hline 2 & 37.12 & 21.16 & 18.50 \\
\hline 3 & 17.31 & 11.51 & 8.45 \\
\hline 4 (coarsest) & 7.84 & 6.24 & 3.40 \\
\hline \multicolumn{2}{r|}{ (a) OracleShrint }
\end{tabular}

\begin{tabular}{l|r|r|r}
\hline \multicolumn{1}{c||}{} & \multicolumn{3}{c}{ Orientations } \\
\hline Scales & HH & HL & LH \\
\hline \hline 1 (finest) & $(95.66)$ & $(95.66)$ & $(95.66)$ \\
\hline 2 & 89.48 & 21.78 & 18.86 \\
\hline 3 & 18.52 & 11.46 & 9.14 \\
\hline 4 (coarsest) & 8.44 & 5.58 & 3.56 \\
\hline
\end{tabular}

(b) Sureshrink: SURE or (universal)

\begin{tabular}{l|r|r|r}
\hline \multicolumn{1}{l||}{} & \multicolumn{3}{c}{ Orientations } \\
\hline Scales & HH & HI & LH \\
\hline \hline 1 (finest) & $(95.85)$ & 49.69 & 51.34 \\
\hline 2 & 46.37 & 19.78 & 16.84 \\
\hline 3 & 17.43 & 8.45 & 6.91 \\
\hline 4 (coarsest) & 7.03 & 2.86 & 3.13 \\
\hline \multicolumn{2}{r|}{ (c) BayesShrink $T_{B}$}
\end{tabular}

Fig.6. The Threshold values of OracleShrink, SureShrink, And BayesShrink,

noise variances, to locate near-optimal Parent subtrees that lie among the best domain subtrees in terms of collage distance.The proposed BayesShrink threshold specifies the zero-zone of the quantization step of this coder, and this zero-zone is the main agent in the coder which removes the noise. Although the setting in this paper was in the wavelet domain, the idea can be extended to other transform domains such as DCT, which also relies on the energy compaction and sparse representation properties to achieve good compression. 
The International journal of Multimedia \& Its Applications (IJMA) Vol.2, No.3, August 2010

\section{REFERENCES}

[1] M. Vetterli, "Wavelets, approximation and compression," IEEE Signal Process. Mag., no. 9, pp. 5973, Sep. 2001.

[2] E. Candes and D. Donoho, "New tight frames of curvelets and optimal representations of objects with smooth singularities," Tech. Rep., Stanford Univ., Stanford, CA, 2002.

[3] M. Do and M. Vetterli, "Pyramidal directional filter banks and curvelets," in Proc. IEEE Int. Conf. Image Processing, Oct. 2001, vol. 3, pp. 158161.

[4] M. N. Do and M. Vetterli, "The contourlet transform: An efficient directional multiresolution image representation,” IEEE Trans. Image Process., vol. 14, no. 12, pp. 20912106, Dec. 2005.

[5] Y. Lu and M. N. Do, "Crisp-contourlets: A critically sampled directional multiresolution image representation," presented at the SPIE Conf. Wavelet Applications in Signal and Image Processing Aug. 2003.

[6] E. P. Simoncelli and W. T. Freeman, “The steerable pyramid: Aflexiblearchitecture for multi-scale derivative computation, in Proc. IEEE Int. Conf. Image Processing, Oct. 1995, vol. 3, pp. 444447.

[7] N. Kingsbury and T. Reeves, "Iterative image coding with overcomplete complex wavelet transforms, SPIE Vis. Commun. Image Process., vol. 5150, pp. 12531264, Jul. 2003.

[8] P. Perona and J. Malik, "Scale-space and edge detection using anisotropic diffusion," IEEE Trans. Pattern Anal. Mach. Intell., vol. 12, no. 7, pp. 629-639, Jul. 1990.

[9] P. Mrazek, J. Weickert, and G. Steidl, "Diffusion inspired shrinkage functions and stability results for wavelet denoising,” Int. J. Comput. Vis., vol. 64, pp. 171-186, 2005.

[10] J. Ma and G. Plonka, “Combined curvelet shrinkage and nonlinear anisotropic diffusion,” IEEE Trans. On Image Processing, vol. 16, no.9, pp. 2198-2206, Sep. 2007.

[11] A. Jacquin, "Image coding based on a fractal theory of iterated contractive image transformations", IEEE Trans. Image Processing, vol. 1, pp. 18-30, 1992.

[12] M. Ghazel, G. Freeman and E.R. Vrscay, "Fractal image denoising", IEEE Trans. Image Processing, vol. 12, no. 12, pp. 1560-1578, 2003.

[13] M. Ghazel, Adaptive Fractal and Wavelet Image Denoising, Ph.D. thesis, ECE Dept., University of Waterloo, May 2004.

[14] A. Jacquin, "Image coding based on a fractal theory of iterated contractive image transformations", IEEE Trans. Image Processing, vol. 1, pp.18-30, 1992.

[15] N. Lu, Fractal Imaging, New York: Academic Press, 1997.

[16] G. Davis, “A wavelet-based analysis fractal image compression,” IEEE Trans. Image Process., vol. 7, no. 2, pp. 141154, Feb. 1998.

[17] B. Forte and E. R. Vrscay, "Inverse problem methods for generalized fractal transforms," in Fractal Image Encoding and Analysis, ser. NATO ASI F 159, Y. Fisher, Ed. New York: Springer-Verlag, 1998.

[18] H. Krupnik, D. Malah, and E. Karnin, "Fractal representation of images via the discrete wavelet transform,” presented at the IEEE Conv. Elect. Eng. Tel-Aviv, Israel, Mar. 79, 1995. 


\section{Authors}

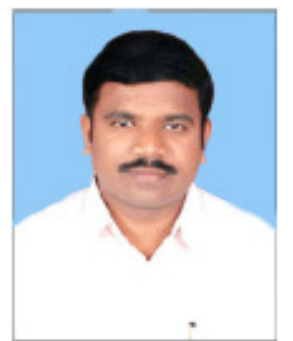

B. Chinna Rao is currently pursuing Ph.D from JNTU. He has been actively guiding students in the area of Signal and Image Processing. He has published 2 international journals and 6 International Conferences. Currently, he has been working as Associate Professor in Dept of Electronics and Communication Engineering, RK College of Engineering, Ibrahimpatnam, Vijayawada, Andhra Pradesh, India.

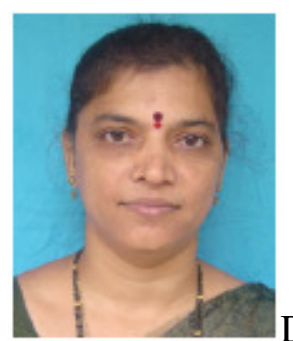

Dr. M. Madhavi Latha is specialized in signal and image processing using wavelets and Low Power Mixed Signal Design in VLSI. She has published 30 publications in various journals and conferences at national and International level and presented papers in conferences held at Lasvegas, Louisiana, USA and Iunstruck, Austria presently guiding Six students for Ph.D and one student for M.S by research. At present, working as professor of ECE, JNTU College of Engineering, Hyderabad, India. 